Universidade Tecnológica Federal do Paraná - UTFPR

Campus Ponta Grossa - Paraná - Brasil

ISSN: 1981-3686 / v. 07, n. 02: p. 106-1073, 2013

D.O.I: $10.3895 / \mathrm{S} 1981-36862013000200006$
Revista Brasileira deTecnologia

Agroindustrial

\title{
SUBSTITUIÇÃO DE SACAROSE POR FRUTOOLIGOSACARÍDEO EM SORVETE
}

\section{SUBSTITUTION OF SUGAR IN ICE CREAM BY FRUCTOOLIGOSACCHARIDES}

\author{
Vanessa Jurca Seolin ${ }^{1}$; Monica Regina da Silva Scapim²; Gabriella Giani Pieretti ${ }^{3}$, Lucineia Aparecida \\ Cestari Tonon ${ }^{4}$; Grasiele Scaramal Madrona ${ }^{5}$. \\ 1,2,3 Universidade Estadual de Maringá - UEM - Maringá, vanessa_jurca_s@ @ hotmail.com, \\ jprsgrss@yahoo.com.br, ggpieretti@gmail.com, lucestari@gmail.com,gsmadrona@uem.br
}

\begin{abstract}
Resumo
O avanço dos conhecimentos sobre a relação entre alimentação e saúde, bem como os elevados custos da saúde pública e a busca permanente da indústria por inovações têm gerado novos produtos, cujas funções pretendem ir além do conhecido papel nutricional dos alimentos. Pesquisas têm sido realizadas visando à identificação de novos compostos bioativos e o estabelecimento de bases científicas para a comprovação das alegações de propriedades funcionais dos alimentos. Neste contexto, surge uma alternativa para substituição do açúcar (ingrediente restrito a pessoas diabéticas) os frutooligossacarídeos (FOS). O produto escolhido foi o sorvete devido ao seu valor nutritivo e de ser um produto com aceitação sensorial em todas as faixas etárias, foram elaboradas duas formulações com diferentes concentrações de FOS (50\% e 100\% de substituição). Realizou-se análise sensorial com escala hedônica modificada com 150 provadores avaliando os parâmetros cor, sabor, aroma e textura. Também foram realizadas análises microbiológicas e físico-químicas. Os dados foram avaliados por ANOVA e teste de Tukey ao nível de $5 \%$ de significância. Os resultados mostraram que há diferença significativa sensorialmente entre as amostras, sendo que a amostra com 50\% sacarose e 50\% FOS apresentou as maiores médias para todos os atributos avaliados (cor, sabor, aroma e textura). Assim, os produtos desenvolvidos estão dentro dos padrões de legislação estabelecidos em relação às características fisico-quimicas e microbiológicas.
\end{abstract}

Palavras-chave: cappuccino; análise sensorial; textura.

\section{Introdução}

Gelados comestíveis são definidos como produtos alimentícios obtidos a partir de uma emulsão de gorduras e proteínas, com ou sem adição de outros ingredientes e substâncias que tenham sido submetidas ao congelamento, em condições que garantam a conservação do produto no estado congelado ou parcialmente congelado, durante o armazenamento, o transporte, a 
comercialização e a entrega ao consumo. Quanto a sua classificação os sorvetes, são os produtos elaborados basicamente com leite e ou derivados lácteos e ou gorduras comestíveis, podendo ser adicionado de outros ingredientes alimentares, são misturas homogêneas ou não de ingredientes alimentares, batidas e resfriadas até o congelamento, resultando em massa aerada (BRASIL, 2013).

Dados da Associação Brasileira das Indústrias de Sorvete (ABIS) mostram que o mercado sorveteiro está em destaque. Entre 2002 e 2009, consumo total de sorvetes no Brasil cresceu 39,5\%, passando de 713 milhões de litros/ano para 995 milhões de litros/ano (ABIS, 2011). O sorvete é um alimento universal e nutritivo. Ainda segundo dados da ABIS, a cada dez pessoas, nove gostam de sorvetes, que são indicados para todas as idades e seu consumo na última década, obteve aumento de mais de $76 \%$.

Segundo Carvalho et al. (2006) o setor tem buscado saídas criativas para motivar o consumo como o desenvolvimento de novos produtos ou a adoção de matérias prima que apresentem vantagens únicas favorecendo a diferenciação.

O avanço dos conhecimentos sobre a relação entre alimentação e saúde, bem como os elevados custos da saúde pública e a busca permanente da indústria por inovações têm gerado novos produtos, cujas funções pretendem ir além do conhecido papel nutricional dos alimentos. Pesquisas têm sido realizadas visando à identificação de novos compostos bioativos e o estabelecimento de bases científicas para a comprovação das alegações de propriedades funcionais dos alimentos.

O mercado para esse tipo de alimento movimenta cerca de US\$ 60 bilhões no mundo, responsável por mais da metade dos investimentos publicitários na área alimentícia e com expectativas de crescimento da ordem de $5 \%$ ao ano. Trata-se, portanto, de um segmento de grande interesse para as indústrias alimentícias, que têm buscado explorar a relação entre o consumo de determinados ingredientes com a redução de fatores de risco para doenças específicas, principalmente as crônicas não transmissíveis, ou a melhora do desempenho físico ou mental (SALES et al.,2008).

Neste contexto, surge uma alternativa para substituição do açúcar/sacarose (ingrediente restrito a pessoas diabéticas) que são os frutooligossacarídeos (FOS).

Os FOS são açúcares não convencionais, não metabolizados pelo organismo humano e não calóricos. São considerados prebióticos uma vez que promovem seletivamente o crescimento de probióticos como Acidophillus e Bifidus. Essa característica faz com que os FOS promovam uma série de benefícios à saúde humana, desde a redução de colesterol sérico até o auxílio na prevenção de alguns tipos de câncer. Este trabalho de revisão realizado pelos autores, aborda principalmente os efeitos da ingestão de FOS na saúde humana, e algumas de suas aplicações, principalmente na indústria de alimentos (PASSOS e PARK, 2003). 
Além das propriedades fisiológicas, as fibras alimentares, especialmente as solúveis ou viscosas, dentre elas inulina e FOS, possuem características que permitem sua aplicação em diversas áreas, pois apresentam elevado poder adoçante e solubilidade, não cristalizam, não precipitam e nem deixam sensação de secura ou areia na boca, não são degradadas durante a maioria dos processos de aquecimento e não são calóricas (VASCONCELOS, 2010).

Como status legal, os FOS são considerados ingredientes e não aditivos alimentares, na maioria dos países. São fibras dietéticas, confirmado pelas autoridades legais em vários países, e nos Estados Unidos possuem o status GRAS (Generally recognized as safe). A sua ingestão pode estar associada à flatulência, e isto se torna mais flagrante em indivíduos que possuem intolerância à lactose. A gravidade desse tipo de sintoma está associada à dose de FOS consumida, isto é, quanto menos FOS, menos sintomas. A ingestão de $20-30 \mathrm{~g}$ por dia geralmente desencadeia o início de um desconforto severo no indivíduo, sendo o ideal seguir as doses recomendadas de cerca de $10 \mathrm{~g}$ por dia. Comercialmente, os FOS são suplementos caros, a cerca de U\$ 0,20 por grama, o consumo nas doses recomendadas pode custar U\$2,00 por dia (SALES et al., 2008).

Ainda segundo os autores, devido as suas características, os FOS podem ser usados em formulações de sorvetes e sobremesas lácteas que levem no rótulo açúcar reduzido, sem adição de açúcar, calorias reduzidas, produto sem açúcar, entre outros, em formulações para diabéticos, em produtos funcionais que promovam efeito nutricional adicional nas áreas de prebióticos, simbióticos, fibras dietéticas, em iogurtes, promovendo efeito simbiótico (além do próprio efeito probiótico do iogurte), em biscoitos e produtos de panificação, substituindo carboidratos e gerando produtos de teor reduzido de açúcar, produtos para diabéticos, entre outros, em barras de cereais, sucos e néctares frescos, produtos de confeitaria, molhos, entre outros (SALES et al., 2008).

Os FOS possuem características que permitem sua aplicação em várias áreas. Como apresentam cerca de um terço do poder adoçante da sacarose e não são calóricos, não podem ser considerados carboidratos ou açúcares nem fonte de energia, mas podem ser usados de modo seguro por diabéticos. Têm solubilidade maior que a da sacarose, não cristalizam, não precipitam, e nem deixam sensação de secura ou areia na boca. Os FOS podem ser hidrolisados em frutose em condições muito ácidas e em condições de exposição prolongada de determinados binômios tempo / temperatura (BORNET, 1994 e YUN, 1996).

O crescente aumento da busca no mercado por produtos que apresentem além de qualidade sensorial, nutricional e benefícios associados à saúde, faz com que as pesquisas nesse ramo cresçam. Assim, o objetivo desse trabalho foi desenvolver um sorvete com substituição total ou parcial de açúcar por FOS, elaborando assim, um produto saudável sem perder características sensoriais. 


\section{Material e Métodos}

\section{Material}

Os materiais utilizados foram Leite UHT (Parmalatß), nata (Lider®) e açúcar cristal (União®) adquiridos no comércio local. Os outros insumos, como estabilizante e emulsificante, foram doados pela Empresa Duas Rodas, o FOS foi doado pela empresa Clariant, para o desenvolvimento da presente pesquisa.

\section{Metodologia}

A etapa da produção do sorvete foi executada no laboratório de Tecnologia de leite da Universidade Estadual de Maringá, sendo que a calda base foi pasteurizada a $72{ }^{\circ} \mathrm{C}$, por 15 minutos e logo após ocorreo o congelamento em 30 minutos a $-18{ }^{\circ} \mathrm{C}$ em uma produtora descontínua de sorvete. Foram preparadas duas formulações de sorvete sabor capuccino (Tabela 1), sendo a amostra A 50\% de substituição do açúcar por FOS e amostra B com 100\%.

Tabela 1 - Formulações estudadas utilizando diferentes concentrações de FOS

\begin{tabular}{l|rc}
\hline Ingredientes & Amostra $\boldsymbol{A}$ & Amostra B \\
\hline Leite & $69,39 \%$ & $69,39 \%$ \\
Cappuccino & $13,61 \%$ & $13,61 \%$ \\
Açúcar & $6,80 \%$ & - \\
FOS & $6,80 \%$ & $13,60 \%$ \\
Nata & $2,04 \%$ & $2,04 \%$ \\
Liga neutra & $0,68 \%$ & $0,68 \%$ \\
Emulsificante & $0,68 \%$ & $0,68 \%$ \\
\hline
\end{tabular}

Rendimento do sorvete (overrun)

A determinação do overrun foi por meio da massa do sorvete antes do processo de congelamento e areação e após esse processo. O cálculo foi feito conforme a equação abaixo (Sabatini et al, 2011).

$\%$ overrun $=\frac{(\mathrm{P} 1-\mathrm{P} 2)}{\mathrm{P} 2} \times 100$

Onde:

P1 é o produto aerado.

P2 é o produto derretido. 
As análises de composição centesimal foram realizadas logo após a produção das diferentes formulações de sorvete. Foram realizadas análises de fibra bruta, proteína, lipídios, cinzas e umidade seguindo a metodologia do Adolfo Lutz (IAL, 2005). As análises foram realizadas em triplicata.

\section{Análise Instrumental de textura}

A análise foi realizada em um Texturômetro modelo Stable Micro Systems Texture Analyser TAXT2 (Texture Technologies Corp, Inglaterra). As características do ensaio foram: acessório Probe 36mm; Força medida em compressão; Opção: retornar ao início; Velocidade de pré-teste: 2,0mm/s; Velocidade de teste: 3,0 mm/s; Velocidade de pós-teste: 7,0mm/s; Distância: 10mm.

Foram realizadas oito leituras para cada amostra e a média do resultado foi apresentada na forma força (kgf) para compressão da amostra.

\section{Análise Sensorial}

Os testes foram aplicados no Laboratório de Análise Sensorial de Alimentos do curso de Engenharia de Alimentos da UEM. As cabines, individuais, equipadas com cadeiras e mesas devidamente projetadas, iluminadas por luz branca. Foi realizada com 150 provadores voluntários, não treinados, de ambos os sexos e sem idade pré-determinada. Foi utilizada escala hedônica modificada de 0-100 ( $0=$ desgostei muito mais que imaginava e $100=$ gostei muito mais que imaginava) (SCHUTZ e CARDELL, 2001), conforme Figura 1. Esta escala apresenta números e palavras correspondentes não equidistantes, uma alternativa a escala hedônica tradicional.

Figura 1 - Escala Hedônica Modificada (tradução de SCHUTZ e CARDELL, 2001)

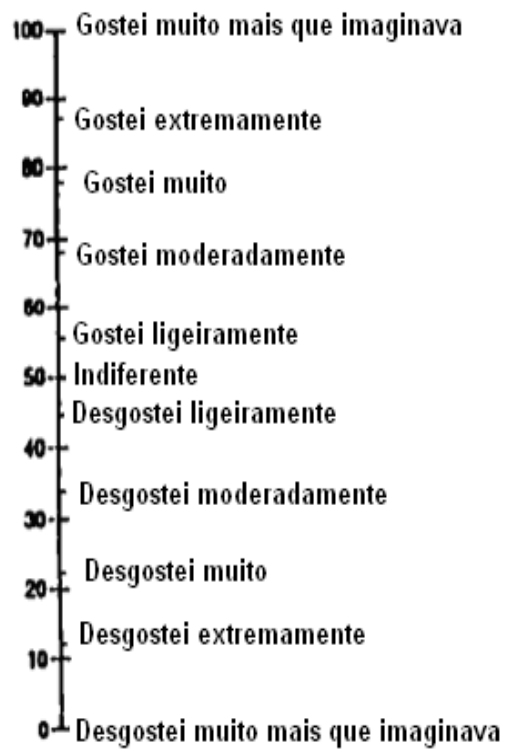


Foram avaliados os atributos cor, aroma, sabor, textura. Os provadores receberam aproximadamente 20 gramas de cada amostra, em temperatura variando entre -10 e $-8^{\circ} \mathrm{C}$, em copos de plástico descartáveis com capacidade para $50 \mathrm{~mL}$, codificados com números aleatórios de três dígitos.

Os provadores receberam as amostras junto a um copo de água para a limpeza do palato entre as amostras, uma ficha sensorial e o Termo de Consentimento Livre e Esclarecido. Este projeto foi aprovado pelo Comitê de ética e pesquisa em seres humanos, conforme protocolo $\mathrm{n}^{\circ}$ 03012912.9.0000.0104.

\section{Análises Microbiológicas}

Foram realizadas logo após a fabricação as análises exigidas pela ANVISA (Resolução RDC $\mathrm{n}^{\mathrm{o}}$ 12, de 2 de janeiro de 2001) (BRASIL, 2011), contagem de coliformes termotolerantes, Contagem de Staphylococcus aureus e Salmonella. As análises foram realizadas em triplicata, seguindo o Método da Instrução Normativa No 62/2003 - MAPA.

\section{Análise estatística dos dados}

Os dados obtidos foram avaliados pelo método de análise de variância (ANOVA) e em caso de diferença significativa entre as amostras a um nível de 5\% de significância o parâmetro foi avaliado pelo teste de Tukey, utilizando planilhas do Microsoft Office EXCEL 2007.

\section{Resultados e discussão}

\section{Overrun}

O overrun é a quantidade de ar incorporado no sorvete através do batimento. Obteve-se uma porcentagem de overrun de 14,85\% para amostra A e de 1,81\% para amostra B. Observou-se que para a amostra com substituição total de açúcar houve pouca incorporação de ar, isso pode ter ocorrido devido às propriedades do FOS e sua granulometria ser menor que a do açúcar adicionado.

A incorporação de ar está diretamente ligada ao tipo de matéria-prima utilizada na fabricação do produto, como é o caso do teor de gordura. Karaca et al. (2009) avaliaram a redução de gordura em diferentes níveis em sorvetes e obtiveram valores de overrun entre 10 e 58\%, segundo os autores quanto menor a quantidade de gordura menor o overrun.

\section{Análise físico-química e de textura}

A Tabela 2 apresenta os resultados das análises físico-químicas das amostras avaliadas. 
Tabela 2 - Resultados da caracterização físico-química das formulações de sorvete com 50 e $100 \%$ de substituição de

\begin{tabular}{lccc}
\multicolumn{5}{c}{ sacarose por FOS } & Legislação \\
\hline Análises & Amostra A & Amostra B & Mínimo 2,5 \\
\hline Proteína (\%) & $5,68^{\mathrm{a}} \pm(0,015)$ & $5,76^{\mathrm{a}} \pm(0,001)$ & Mínimo 2,5 \\
Lipídios (\%) & $5,20^{\mathrm{a}} \pm(0,005)$ & $5,45^{\mathrm{a}} \pm(0,010)$ & - \\
Umidade (\%) & $70,27^{\mathrm{a}} \pm(1,209)$ & $70,73^{\mathrm{a}} \pm(0,169)$ & - \\
Cinzas (\%) & $0,44^{\mathrm{a}} \pm(0,014)$ & $0,45^{\mathrm{a}} \pm(0,035)$ & - \\
Textura (Kgf) & $1089,03^{\mathrm{a}} \pm(0,115)$ & $1137,43^{\mathrm{a}} \pm(0,547)$ & \\
\hline *Letras iguais na mesma linha representam resultados estatisticamente iguais pelo teste de Tukey $(\mathrm{p}<0,05)$. &
\end{tabular}

Não houve diferença significativa entre as amostras $(\mathrm{p}<0,05)$ para nenhum dos parâmetros avaliados. O padrão de identidade e qualidade de sorvetes limita que o valor mínimo de lipídio e proteína presente deve ser de 2,5g/100g. As duas formulações estão dentro do padrão estabelecido.

Pereira et al. (2011) avaliaram a influência da substituição parcial do leite por extrato de soja em sorvete e encontraram (para a amostra de $100 \%$ leite) valores de cinzas de $0,94 \%$ maior que os valores encontrados neste trabalho. O teor de proteína encontrado pelos autores $(3,99 \%)$ foi menor e o de gordura $(10,15 \%)$ maior em relação aos valores encontrados no presente trabalho. Esta diferença pode ser explicada pelo tipo de leite utilizado, que no caso dos autores foi leite em pó desnatado. E ainda pela formulação utilizada, por exemplo, a porcentagem de gordura no presente trabalho foi de $2 \%$ e os autores utilizaram $10 \%$.

O teor de fibra bruta não foi detectado em nenhuma das amostras, provavelmente devido à fibra presente no FOS ser a alimentar e não a bruta. Segundo Lemos (2008) o FOS é confirmado como fibra alimentar ou dietética pelas autoridades legais em vários países e nos Estados Unidos como dito anteriormente leva o status de GRAS.

A textura, medida na forma de pico da força necessária para cisalhar a amostra, indica a rigidez estrutural do produto: quanto mais firme a amostra maior a força necessária para cisalhá-la (SILVA e BOLINI, 2006). Observou-se que não há diferença entre as amostras em relação à textura. No trabalho de Silva e Bolini (2006) foram realizadas análises de textura para sorvetes com adição de soro de queijo. O sorvete com característica próxima ao do presente trabalho foi o padrão sem substituição de leite por soro, com firmeza de $4699 \mathrm{Kgf} / \mathrm{s}$.

No sorvete sabor cappuccino, desenvolvido neste trabalho, existe a adição de leite em pó dando uma característica de maior maciez e conseqüentemente menor resistência ao cisalhamento. Segundo Souza et al. (2010) a textura está relacionada ao conteúdo proteico, sendo que um alto conteúdo de proteína permite a obtenção de um produto com melhor textura, maior facilidade de batimento e maior rendimento. 


\section{Análise sensorial}

Segundo Guinard (1998) o sorvete oferece uma combinação de propriedades sensoriais altamente desejáveis sendo estas classificadas em categorias de aparência (cor, maciez, regularidade), aroma, sabor e textura/preenchimento bucal (dureza, viscosidade, cremosidade). A Tabela 3 apresenta os resultados da análise sensorial e a Figura 2 as médias recebidas para cada atributo.

Tabela 3 - Notas atribuídas às amostras pelos provadores na escala hedônica modificada

\begin{tabular}{lll}
\hline Atributos sensoriais & Amostra A & Amostra B \\
\hline Cor & $77,54 \mathrm{a}$ & $74,69 \mathrm{~b}$ \\
Aroma & $74,45 \mathrm{a}$ & $68,06 \mathrm{~b}$ \\
Textura & $80,40 \mathrm{a}$ & $72,99 \mathrm{~b}$ \\
Sabor & $80,60 \mathrm{a}$ & $61,63 \mathrm{~b}$ \\
\hline
\end{tabular}

* Letras diferentes na mesma linha indicam diferença significativa $(p<0,05)$

Figura 2 - Média das notas atribuídas pelos provadores para os atributos cor, sabor, aroma e textura por meio da escala hedônica modificada

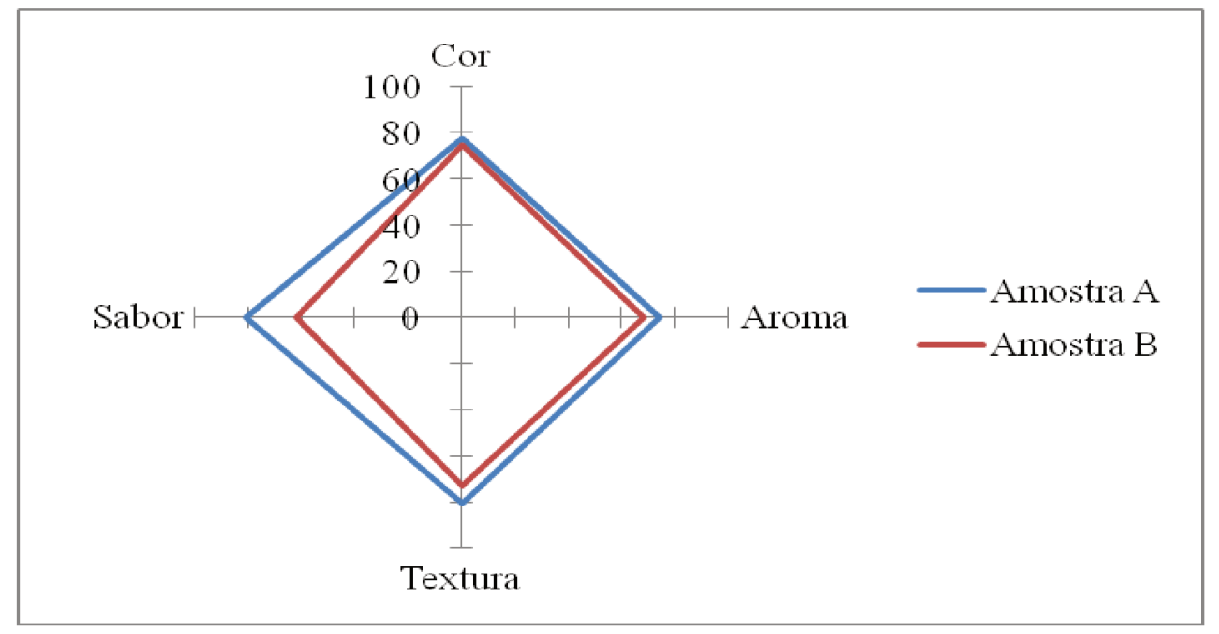

Houve diferença significativa em todos os parâmetros ao nível de significância de 5\%, porem as médias das notas atribuídas consideraram-se satisfatórias. Em todos os parâmetros avaliados a aceitação do sorvete com substituição de $50 \%$ de FOS (Amostra A) foi maior. Em todos os parâmetros a aceitação para as amostras ficaram entre "gostei ligeiramente" e "gostei extremamente".

Sabe-se que o consumo de sorvete está mais ligado ao prazer, do que ao seu próprio valor nutricional. Assim, vários estudos têm sido realizados adicionando outros ingredientes, com intuito de melhorar esta qualidade nutricional do sorvete, conforme os trabalhos citados abaixo. 
Silva e Bolini (2006) avaliaram sensorialmente um sorvete produzido com diferentes concentrações de soro e observaram que os produtos formulados com substituição do leite em pó desnatado por $30 \%$ soro ácido em pó ou $100 \%$ soro desmineralizado ou 100\% CPS-35(concentrado protéico de soro 35\%), tiveram tão boa aceitação sensorial quanto o padrão formulado apenas com leite em pó desnatado.

Shaviklo et al. (2011) fortificaram sorvete com proteína de peixe e encontraram que concentrações de 30 e $5 \mathrm{~g} / \mathrm{Kg}$ de proteína não influenciaram nos atributos sensoriais avaliados e ainda aumentaram o teor de proteína dos sorvetes, sendo possível este tipo de fortificação.

Wood (2011) sugeriu que FOS podem ser adicionados como ingrediente em sorvete em substituição de sacarose em até $20 \%$, pois em maiores concentrações pode ocorrer alteração nas características sensoriais e diminuição da aceitação do produto, conforme também foi observado no presente trabalho.

Sales et al, (2008) avaliaram sensorialmente formulações de sorvete com adição de granola e FOS e obtiveram para aceitação global que a maior média de julgamentos foi para o sorvete adicionado de FOS $(7,82)$, que não diferiu estatisticamente das amostras controle $(7,53)$ e contendo FOS e granola $(7,41)$. No entanto, o sorvete contendo somente granola apresentou menor média de julgamentos $(7,23)$, diferindo significativamente do sorvete contendo FOS.

\section{Análise microbiológica}

A Tabela 3 apresenta os resultados das análises microbiológicas para as amostras A e B.

Tabela 3 - Dados obtidos na análise microbiológica da Amostra A e B (50\% e 100\% FOS)

\begin{tabular}{lccc}
\hline Parâmetros & Amostra A & Amostra B & VPM $^{*}$ \\
\hline Contagem de coliformes termotolerantes (UFC/g) & $<1,0 \times 10^{1}$ & $<1,0 \times 10^{1}$ & $5,0 \times 10^{1}$ \\
Contagem de Staphylococcus aureus (UFC/g) & $<1,0 \times 10^{2}$ & $<1,0 \times 10^{2}$ & $5,0 \times 10^{2}$ \\
Salmonella (em 25 g) & Ausência & Ausência & Ausência \\
\hline VPM: Valor máximo permitido. RDC n 12 (Anvisa, 2012) & & &
\end{tabular}

Os resultados encontram-se dentro dos limites da legislação vigente, sendo assim são satisfatórios, pois se utilizou matéria-prima de excelente qualidade e seguiram-se as normas de higiene. Devido à sua composição (gordura, leite, açúcares, estabilizantes, emulsificantes, aromatizantes, corantes e outros) o sorvete é um alimento propício para o desenvolvimento de microrganismos (OKURA et al., 2009). No entanto, a contaminação só ocorre em casos de negligência nos procedimentos de produção.

De acordo com Falcão et al. (1983) vários são os relatos dos serviços de Saúde Pública dos Estados Unidos e Canadá sobre casos de toxinfecções por microrganismos diversos, principalmente salmonelas, ligados à ingestão de sorvetes. 
Valores superiores de coliformes a $35^{\circ} \mathrm{C}$ e a $45^{\circ} \mathrm{C}$ foram encontrados por Pereira et al., (2009) em um estudo que determinou a qualidade microbiológica de sorvetes comercializados na cidade de Alfenas - MG, com concentrações de $2,4 \times 10^{2} \mathrm{UFC} / \mathrm{g}$ de coliformes à $35^{\circ} \mathrm{C}$ e $1,1 \times 10^{3}$ $\mathrm{UFC} / \mathrm{g}$ de coliformes à $45^{\circ} \mathrm{C}$ ou termotolerantes.

\title{
4 Conclusões
}

O produto desenvolvido apresentou características físico-químicas e microbiológicas dentro dos padrões estabelecidos pela legislação brasileira vigente. Ainda, houve boa aceitação sensorial para as formulações, porém a amostra mais aceita foi a amostra A com substituição de $50 \%$ de sacarose por FOS em todos os parâmetros avaliados. A formulação com 100\% FOS não obteve boa aceitação. Assim, conclui-se que é viável a substituição da sacarose por FOS no sorvete em até $50 \%$.

\begin{abstract}
Advancing knowledge about the relationship between diet and health, and the high costs of public health and the constant search for innovations in the industry have generated new products, whose functions intended to go beyond the known role of nutritional food. Research has been conducted in order to identify new bioactive compounds and the establishment of a scientific basis to prove the allegations of functional properties of food. In this context there is an alternative to replace sugar (an ingredient restricted to people with diabetes) the fructooligosaccharides (FOS). The product chosen was the ice cream because of its nutritional value and be a product with sensory acceptance in allage groups, two formulationswere prepared with different concentrations of FOS. Sensory analysis was performed with modified hedonic scale with 150 tasters evaluating the parameters color, flavor, aroma and texture. Were analyzed for microbiological and physical-chemical properties. Data were evaluated by ANOVA and Tukey's test at 5\% significance level. The results showed no significant difference between the sensory samples, and the sample with 50\% sucrose and 50\% FOS had the highest means for all attributes (color, flavor, aroma and texture). Thus, the products developed are within the standards established law in relation to physico-chemical and microbiological.
\end{abstract}

Key-words: cappuccino, sensory analysis; texture.

\section{Referências}

ABIS - Associação Brasileira das Indústrias de Sorvete. Consumo mundial. Disponível em<www.fispal.com.br/noticia.asp?cod_noticia=2033\&codevento=1\&codnucleo=1\&cod_menutop=1>, Acesso em: 22 de março de 2011.

BORNET, F. R. Undigestible sugars in food products. American Journal Clinical Nutrition, Paris, v. 59, n. 3, Suppl, p. 763-769, 1994. 
BRASIL, Ministério da Saúde do, Agência Nacional de Vigilância Sanitária. Regulamento Técnico referente a gelados comestíveis, preparados, pós para preparo e bases para gelados comestíveis. Portaria n³79, de 26 de abril de 1999. Disponível em: http://www.anvisa.gov.br/. Acesso em 09/06/2013.

CARvalho, E. A.; MElO, B. N. A.; Aguiar, J. C.; CAldAS, M. C. S.; CAVAlCANTI, M. T.; MIYAJI, M. Desenvolvimento e Análise Sensorial de Sorvete de Massa Sabor Café. I JORNADA NACIONAL DA AGROINDÚSTRIA ,Bananeiras, 17 a 20 de outubro de 2006.

Brasil. Agência Nacional da Vigilância Sanitária. Disponível em: http://www.anvisa.gov.br/legis/resol/12_01rdc.htm. Acessado em: 26/06/2011.

FALCÃO, D. P.; FILHO, G. S.; NISHIDA, N. K.; BORGES, S. R. Exame Mibrobiológico de Sorvetes não Pasteurizados. Revista Saúde Pública, v. 17, p. 2-8, 1983. http://dx.doi.org/10.1590/S0034-89101983000100002

GUINARD, J.X. Session III: Additives, colours, flavours. In: BUCHHEIM, W. Ice Cream - Proceedings of the international symposium held in Athens. International Dairy Federation, p. 91-103, 1998.

IAL. Normas Analíticas do Instituto Adolfo Lutz. Métodos químicos e físicos para análise de Alimentos. São Paulo: Vol. 1., 4 ed. 2005.

KARACA; O. B.; GÜVEN, M.; YASAR, K.; KAYA, S.; KAHYAOGLU, T. The functional, rheological and sensory characteristics of ice creams with various fat replacers. International Journal of Dairy Technology. v. 62, p. 93-99, 2009. http://dx.doi.org/10.1111/j.1471-0307.2008.00456.x

LEMOS, A.C.G. Efeito da suplementação de frutooligossacarídeos (FOS) sobre o sistema Imunológico: estudo em ratos. Campinas. 2008. 154 f. Dissertação. Mestrado em Ciência de Alimentos. Universidade Estadual de Campinas.

OKURA, M. H; RABELO, T. M; MIGUEL, D. P; FREITAS, M. P. Avaliação microbiológica em amostras de sorvetes, coletadas no município de Uberaba, MG. Revista Higiene Alimentar. v. 23, n. 172/173, p. 166-170, 2009.

PASSOS, L. M. L., PARK, Y. K., Frutooligossacarídeos: implicações na saúde humana e utilização em alimentos, Ciencia Rural, v. 33, n. 2, 2003. http://dx.doi.org/10.1590/S0103-84782003000200034

PEREIRA, G. G.; RESENDE, J. V.; ABREU, L. R.; GIAROLA, T. M. O.; PERRONE, I. T. Influence of the partial substitution of skim milk powder for soy extract on ice cream structure and quality. Euro Food Rechear Technology, v. 232, p. 1093-1102. 2011. http://dx.doi.org/10.1007/s00217-011-1483-z

PEREIRA, M. A.; NASCIMENTO, L. C.; FIORINI, J. E. Determinação da qualidade microbiológica de sorvetes comercializados na cidade de Alfenas, MG. Revista Higiene Alimentar, v. 23, n. 168/169, p. 162-165, 2009.

SABATINI, D. R.; SILVA, K. M.; PICININ, M. E.; DEL SANTO, V. R.; SOUZA, G. B.; PEREIRA, C. A. M. Composição centesimal e mineral da alfarroba em pó e sua utilização na elaboração e aceitabilidade em sorvete. Alimentos e Nutrição, v. 22, n. 1, p. 129-136, 2011.

SCHUTZ, H. G., CARDELL, A. V. A labeled affective magnitude (LAM) scale for assessing food liking/disliking. Journal of Sensory Studies, v.16, p.117-159, 2001. http://dx.doi.org/10.1111/j.1745-459X.2001.tb00293.x

SHAVIKLO, G.R.; THORKELSSON, G.; SVEINSDOTTIRC, K.; RAFIPOURB, F. Chemical properties and sensory quality of ice cream fortified with fish protein. Journal Science Food Agriculture; v. 91, p. 1199-1204. 2011. http://dx.doi.org/10.1002/jsfa.4299

SILVA, K., BOLINI H. M. A., Avaliação Sensorial de Sorvete Formulado com Produto de Soro Ácido de Leite Bovino. Ciência e Tecnologia de Alimentos. v. 26, n.1, p. 116-122. 2006. http://dx.doi.org/10.1590/S0101-20612006000100020

SALES, R. L., VOLP, A. C. P., BARBOSA, K. B. F., DANTAS, M. I. S., DUARTE, H. S., MINIM V. P. R. Mapa de preferência de sorvetes ricos em fibras. Ciência e Tecnologia de Alimentos. V. 28, p. 27-31, 2008. http://dx.doi.org/10.1590/S0101-20612008000500005

SOUZA, J. C. B., COSTA, M. R., DE RENSIS, C. M. V., SIVIERI, K. Sorvete: Composição, Processamento e Viabilidade da adição de Probiótico. Alimentos e Nutrição v. 21, n. 1, p. 155-165. 2010. 
VASCONCELOS, C. M.; SILVA, C. O.; TEIXEIRA, L. J. Q.; CHAVES, J. B. P.; MARTINO, H. S. D.; Determinação da fração da fibra alimentar solúvel em raiz e farinha de yacon (Smallanthus sonchifolius) pelo método enzimáticogravimétrico e cromatografia líquida de alta eficiência. Revista do Instuto Adolfo Lutz, v. 69, p. 188-193, 2010.

WOOD, J. M. Sensory evaluation of ice cream made with prebiotic ingredients substituted for sugar. University of Nebraska. Lincoln. 2011.

YUN, J. W. Fructooligosaccharides - Occurrence, preparation and applications. Enzymes and Microbial Technology, Kyungbug, v.19, p.107-117, 1996.

Submetido em 15 fev. 2013, Aceito para publicação em 15 jul. 2013. 